\title{
THE SAFETY AND EFFICACY OF SULFADOXINE-PYRIMETHAMINE, AMODIAQUINE, AND THEIR COMBINATION IN THE TREATMENT OF UNCOMPLICATED PLASMODIUM FALCIPARUM MALARIA
}

\author{
DAVID SCHELLENBERG, ELIZEUS KAHIGWA, CHRIS DRAKELEY, ATHUMANI MALENDE, JOHN WIGAYI, \\ CHRIS MSOKAME, JOHN J. APONTE, MARCEL TANNER, HASSAN MSHINDA, CLARA MENENDEZ, AND \\ PEDRO L. ALONSO \\ Unidad de Epidemiologia, Hospital Clinic, Barcelona, Spain; Ifakara Health Research and Development Centre, Ifakara, Tanzania; \\ St. Francis Designated District Hospital, Ifakara, Tanzania; Swiss Tropical Institute, Basel, Switzerland
}

\begin{abstract}
The safety and efficacy of amodiaquine (AQ), sulfadoxine-pyrimethamine (SP), and coadministered AQ+SP was assessed in 351 Tanzanian children (age range, 6-59 months) with uncomplicated Plasmodium falciparum malaria. This open, randomized study followed the 28-day World Health Organization (WHO) protocol and evaluated safety using clinical and laboratory parameters. Children receiving SP were more likely to vomit during follow-up (32\% vs. $17 \%: P=0.03$ ), and SP alone resulted in prolonged fever clearance times. Although Day 7 and Day 14 clinical and parasitological cure rates were similar, by Day $2845 \%$ of children treated with AQ demonstrated R1 resistance and $27.5 \%$ were clinical failures compared with $25 \%$ and $6.3 \%$, respectively, for SP alone. Coadministered AQ+SP was safe, combined the greater clinical $(96.2 \%)$ and parasitological $(64.2 \%)$ efficacy of SP with the more rapid symptom resolution of AQ, and reduced the incidence of gametocytemia during follow-up (AQ+SP 12.6\% vs. SP 29.9\%; $P=0.001$ ). The level of R1 resistance to SP may herald a rapid decline in its efficacy as SP drug pressure increases. Coadministration of $\mathrm{AQ}+\mathrm{SP}$ may delay this.
\end{abstract}

\section{INTRODUCTION}

Malaria kills approximately 1 million children every year, most of them in sub-Saharan Africa. ${ }^{1}$ The mainstay of malaria control remains early, effective treatment of clinical cases, ${ }^{2}$ yet the effectiveness of this policy is likely to decrease as resistance to many of the available and affordable antimalarial drugs increases. Resistance to chloroquine (CQ), a common first-line treatment for malaria in endemic countries, is widespread and has been associated with an increase in mortality. ${ }^{3}$ However, the decision to change antimalarial treatment guidelines is complex; limited alternatives exist, and the decision to change should take into consideration a large number of variables, including the efficacy, cost, and availability of drugs, the public health impact of resistance, treatment-seeking behavior, and the likely durability of the new policy. The latter is largely determined by the rate at which resistance to the first-line drug develops, itself a function of the mechanisms of resistance.

There is considerable interest in the use of multiple drugs, with different mechanisms of action, for the treatment of malaria cases. ${ }^{4}$ Such combination treatment (CT) is likely to extend the useful lifetime of the constituent drugs by reducing the rate at which resistance develops. The "protection" of the individual drugs is a function of the likelihood of a resistanceconferring mutation arising at two (or more) relevant Plasmodium falciparum gene loci and is very much lower than the chance of a single mutation arising. CT may also have greater efficacy than treatment with the individual drugs. Artemether and its derivatives are advocated for inclusion in antimalarial CT because they quickly reduce the level of parasitemia and hence the parasite pool from which resistant $P$. falciparum may arise. ${ }^{4} \mathrm{~A}$ number of trials are underway combining artemether with existing antimalarials. Although early results are promising, ${ }^{5}$ it is unclear how quickly artemether could be made available at health facilities in sub-Saharan Africa and at what cost.

Sulfadoxine-pyrimethamine (SP) is a readily available, relatively cheap, and effective antimalarial. Tanzania has joined Malawi and Kenya ${ }^{6,7}$ in replacing CQ with SP as firstline treatment of malaria. However, concerns that the long half-life of the drug may lead to a relatively rapid increase in the rate at which resistance develops, ${ }^{8}$ especially in situations in which the drug pressure is high, have been borne out by molecular 9 and in vivo studies ${ }^{10,11}$ and by the short duration of its efficacy in Thailand. ${ }^{12}$ If SP is to be a useful component of CT, it needs to be protected until combinations with artemether are shown to be safe, effective, and available. Even a rational combination of drugs will yield poor results if resistance to one of the component drugs is high.

There has been renewed interest in amodiaquine (AQ) as a malaria treatment in endemic settings. ${ }^{13}$ Because it is a congener of chloroquine, cross-resistance may limit its efficacy as a first-line antimalarial treatment. ${ }^{14}$ Nevertheless, it is being considered as a second-line treatment in some countries. $^{15}$

Anecdotal reports suggest that $\mathrm{CQ}$ and SP have been increasingly coprescribed in areas of high CQ resistance ${ }^{16}$ despite a lack of safety and efficacy data. ${ }^{17}$ If AQ and SP become widely available, it seems likely that these too will be coadministered. Both agents are associated with adverse reactions affecting the skin and bone marrow ${ }^{13,18}$ and it may be that their combined use will produce an unacceptably high rate of adverse events.

Because of the urgent need to protect SP and generate safety data on AQ+SP, we undertook a three-arm, randomized, open-label safety and efficacy study of AQ, SP, and $\mathrm{AQ}+\mathrm{SP}$ in the treatment of uncomplicated P. falciparum malaria in young Tanzanian children.

\section{MATERIALS AND METHODS}

Study area, population, and recruitment. The study was conducted in the malaria holoendemic setting of Ifakara, situated in the rice-growing flood plains of the Kilombero River, southern Tanzania, and described in detail elsewhere. ${ }^{19}$ Children were recruited from the Mother and Child Health $(\mathrm{MCH})$ Clinic of the 380-bed St. Francis Designated District 
Hospital (SFDDH) between May and August 1999. Malaria is the leading cause of pediatric admission and outpatient attendances. A Day-7 parasitological chloroquine failure rate of $65 \%$ has been described in children aged $1-5$ years. ${ }^{20} \mathrm{SP}$ has been available at health facilities for many years as a secondline malaria treatment. AQ has not been readily available, and there are no previous local efficacy estimates.

After verbal consent from the parent, permanent residents of Ifakara between the ages of 6 months and 5 years presenting to the $\mathrm{MCH}$ Clinic with an axillary temperature $\geq 37.5^{\circ} \mathrm{C}$ were screened for $P$. falciparum parasitemia. Those with an asexual $P$. falciparum parasitemia of $\geq 2,000 / \mu \mathrm{L}$ were eligible for enrollment. The trial objective and procedures were explained in Kiswahili, and a series of questions were asked to assess adequate comprehension. Written informed consent was then requested from the guardians of all children before recruitment to the study. Children were examined by a project clinical officer, and demographic and clinical data were recorded. Children were excluded if they were unable to tolerate oral medication or had any signs indicative of severe or complicated malaria. ${ }^{21}$ Known chronic underlying disease and sensitivity to trial medication were additional exclusion criteria.

Randomization, treatment, and follow-up. Children were randomly allocated to receive $\mathrm{AQ}, \mathrm{SP}$, or $\mathrm{AQ}+\mathrm{SP}$ (all from Shelys Pharmaceuticals, P.O. Box 3016, Pugu Rd, Dar es Salaam, Tanzania) according to a computer-generated list of treatments randomly assigned to study numbers in blocks of nine. Envelopes marked with the study number contained a piece of paper with the allocated treatment for that number printed on it. Study numbers were allocated sequentially to children as they presented. Children were issued with an identity card bearing their study number and other identification information.

All antimalarial doses were given by a member of the research team, the first dose at the $\mathrm{MCH}$ Clinic and the second and third doses of AQ at the children's homes. Dosages were administered according to a weight-dosing schedule, which ensured that the minimum dosages received were $25 \mathrm{mg} / \mathrm{kg}$ for AQ (aiming to administer $10 \mathrm{mg} / \mathrm{kg}$ for Doses 1 and 2 and $5 \mathrm{mg} / \mathrm{kg}$ for Dose 3) and $25 \mathrm{mg} / \mathrm{kg}$ of sulfadoxine with 1.25 $\mathrm{mg} / \mathrm{kg}$ of pyrimethamine. A replacement dose was given if vomiting occured within $30 \mathrm{~min}$ of administration, and rescue medication was given if there was vomiting of the replacement dose. All children were given a single dose of paracetamol, $250 \mathrm{mg}, 30 \mathrm{~min}$ before administration of the trial drug, to control fever. Rescue medication consisted of nine doses of quinine sulphate, $30 \mathrm{mg} / \mathrm{kg} / \mathrm{day}$, in line with the Tanzanian National Treatment Guidelines, and was only given to children with symptomatic parasitemia. Children who were parasitemic on Day 28 received one of the alternative treatment regimens, regardless of fever, as recommended by WHO. ${ }^{21}$

Children were visited at home by a trained field worker 1 , $2,3,14$, and 21 days after recruitment. Children were asked to return to the clinic for assessments on Days 7 and 28, and mothers were encouraged to bring their child to the project clinical officer at any time during follow-up if they were concerned about the child's well-being. At all contacts, including admissions to the pediatric ward, standardized forms were completed to facilitate the detection of any adverse events. A symptom report form, based on the National Institutes of Health common toxicity criteria, was completed at routine follow-up visits when the axillary temperature was measured and a blood slide and filter paper sample were collected for later parasitological and molecular analysis, respectively. In addition, capillary blood collected on Days 0 and 28 in a microtainer was used to measure packed cell volume (PCV), total and differential white cell counts, platelets, alanine transaminase (ALT), $\gamma$-glutamyl transferase $(\gamma$-GT), and creatinine as part of the safety assessment. The study protocol was reviewed and approved by the Ifakara Health Research and Development Centre's Scientific and Ethical review committees.

Laboratory techniques. Field's stain of thick blood films was used to screen children before recruitment when 20 highpower fields were examined. Thick films stained with Giemsa were used for definitive parasite counts. A total of 200 thick film fields were screened for non-falciparum Plasmodia, and the number of asexual forms per 200 leukocytes were recorded and converted into parasite density per microliter by assuming an average white cell count of $8,000 / \mu \mathrm{L}$. Gametocytes were counted against 500 leukocytes. Hence, the limit of detection of asexual forms was $40 / \mu \mathrm{L}$ and for gametocytes $16 / \mu \mathrm{L}$. Each slide was read at least twice; a third reading was performed for major discrepancies between the results of the first two readings (either positive/negative discrepancies or a ratio of the readings $<0.66$ or $>1.33$ ). The majority reading for positive/negative discordances or the geometric mean of three positive readings was taken as the definitive result. PCVs were measured using a microhematocrit tube and microcentrifuge (Hawksley, Lancing, UK). Total and differential white cell counts were estimated using a semiautomated Coulter-type counter (Sysmex 800, TOA Medical Electronics Ltd, Kobe, Japan). Alanine transaminase (ALT), $\gamma$-GT, and creatinine concentrations were measured using a multilayer analizer (Vitros 250, Ortho-Clinical Diagnostics, Johnson \& Johnson). Electronic thermometers were used to measure axillary temperature and were calibrated weekly using a water bath and mercury thermometer.

Outcome measures, data management, and statistical methods. The safety profile was evaluated by documenting the numbers and timing of deaths, admissions, and outpatient attendances in study children. Particular attention was paid to skin reactions and evidence of bone marrow suppression. The proportions of children reporting symptoms absent at baseline but developing during the course of follow-up were compared to assess adverse drug effects. The proportion of children with hematological and biochemical parameters falling outside the normal range at Day 28 was also documented. The efficacy of the different regimens in terms of resolution of symptoms present at baseline was assessed by comparing the total duration of symptoms in the different groups. All comparisons were made relative to SP because this is a standard treatment.

Proportions of children with different levels of parasitological resistance are presented at different times during followup. Standard WHO clinical end points were used ${ }^{22}$ : early treatment failure (ETF), defined as the development of danger signs in the presence of parasitemia or severe malaria during follow-up on Days $0,1,2$, or 3 , an axillary temperature $\geq 37.5^{\circ} \mathrm{C}$ on Day 2 with parasitemia greater than Day 0 , axillary temperature $\geq 37.5^{\circ} \mathrm{C}$ on Day 3 with any parasitemia or Day 3 parasitemia $\geq 25 \%$ of Day 0 parasitemia; late treatment failure (LTF), defined as the development of danger 


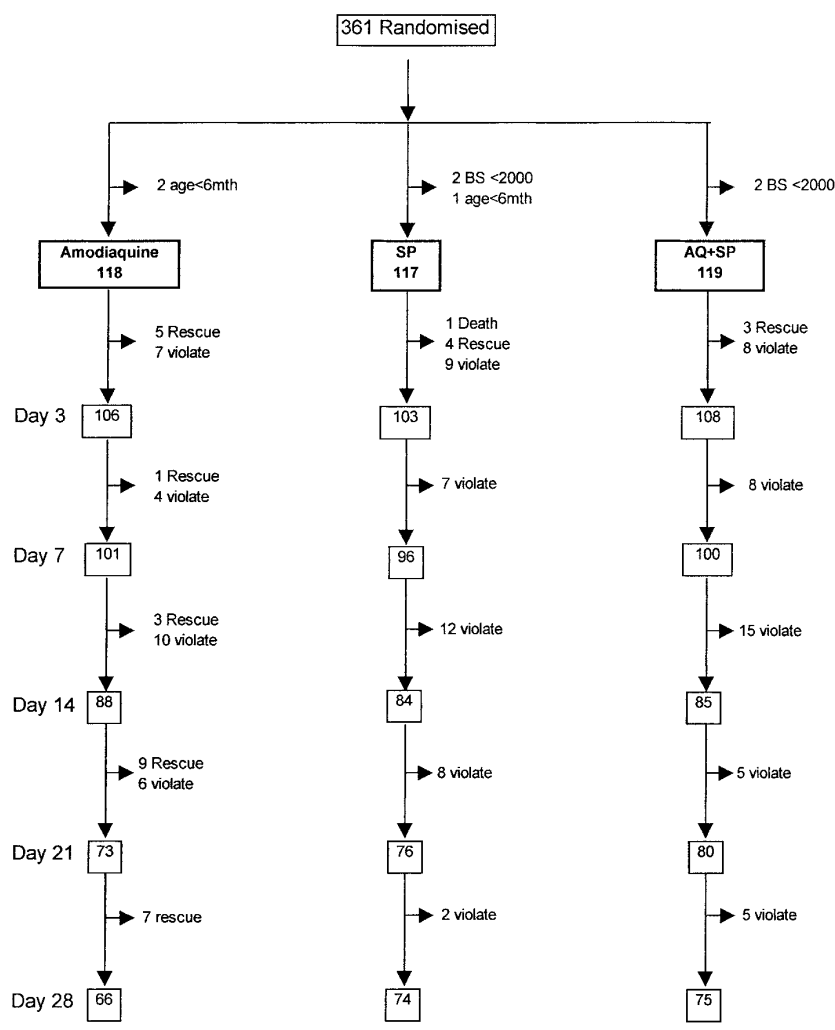

Figure 1. Trial profile. Completeness of follow-up: AQ $(\mathrm{n}=91)$ versus $\mathrm{SP}(\mathrm{n}=79)$ versus $\mathrm{AQ}+\mathrm{SP}(\mathrm{n}=78): \chi^{2}(2)=4.3, P=0.12$. Violate: missed a scheduled contact for reason other than requiring rescue treatment.

signs in the presence of parasitemia or severe malaria during follow-up on Days 4 to Day 28, without prior ETF or a temperature $\geq 37.5^{\circ} \mathrm{C}$ in the presence of parasitemia during follow-up on Days 4 to Day 28 without prior ETF; and adequate treatment response (ATR), defined as the absence of para- sitemia, irrespective of temperature, without previous ETF or LTF or axillary temperature $<37.5^{\circ} \mathrm{C}$, irrespective of parasitemia, without previous ETF or LTF, defined at each time point (i.e., 3, 7, 14, 21, and 28 days).

Data were double entered into FoxPro (version 2.6) databases, and discordances were resolved before range and consistency checks were performed. Analysis was performed using Stata (version 5.0). Dichotomous outcomes were compared using Pearson's chi-square or Fisher's exact test, as appropriate. Mean values of normally distributed variables were compared using Student's $t$ test. This sample size was calculated to estimate failure rates to within $4.3 \%$ of a true failure rate of $5 \%$ and to within $10 \%$ of a true failure rate of $50 \%$.

\section{RESULTS}

A total of 361 children were randomized to receive AQ, SP, or AQ+SP (Figure 1). Three children were subsequently found to be younger than 6 months, and four had definitive parasite counts less than $2000 / \mu \mathrm{L}$. Data from these children were not included in the analyses. Baseline characteristics (Table 1) and completeness of follow-up (see Figure 1) were similar in all groups, although children receiving AQ alone tended to be older than those receiving other treatments. Overall, 35 (9.9\%) children moved to the family farms during follow-up, $56(15.8 \%)$ failed to attend one or more follow-up visits, and $15(4.2 \%)$ were withdrawn for other reasons, including two children who failed to receive a dose of AQ. There were no significant differences in these reasons between the different groups.

Safety (Table 2). A 1-year old girl who received SP became drowsy $6 \mathrm{~h}$ after recruitment and was admitted with an axillary temperature of $38.5^{\circ} \mathrm{C}$, Blantyre coma score of $4 / 5$, and mild dehydration. Convulsions shortly after admission were controlled with diazepam, and she received intravenous quinine. Her condition continued to deteriorate, and she died

TABLE 1

Baseline characteristics

\begin{tabular}{|c|c|c|c|}
\hline \multirow[b]{2}{*}{ Variable } & \multicolumn{3}{|c|}{ Treatment group } \\
\hline & $\mathrm{AQ}(\mathrm{n}=118)$ & $\mathrm{SP}(\mathrm{n}=117)$ & $\mathrm{AQ}+\mathrm{SP}(\mathrm{n}=119)$ \\
\hline No. males $(\%)$ & $47(39.8 \%)$ & $58(49.6 \%)$ & $59(49.6 \%)$ \\
\hline Mean age (years) & $2.51(2.28-2.73)$ & $2.22(2.01-2.43)$ & $2.11(1.89-2.33)$ \\
\hline Mean PCV & $29.2(28.1-30.3)$ & $28.6(27.5-29.7)$ & $29.4(28.3-30.5)$ \\
\hline No. with $\mathrm{PCV}<25 \%$ (\%) & $23(20.0)$ & $27(23.4)$ & $22(18.6)$ \\
\hline Mean temperature $\left({ }^{\circ} \mathrm{C}\right)$ & $38.5(38.3-38.7)$ & $38.7(38.5-38.9)$ & $38.7(38.5-38.9)$ \\
\hline Mean weight $(\mathrm{kg})$ & $11.1(10.7-11.6)$ & $10.6(10.1-11.1)$ & $10.8(10.1-11.4)$ \\
\hline \multicolumn{4}{|c|}{ Parasite density $(\mu \mathrm{L})$ Geometric mean range } \\
\hline Mean & 43,026 & 53,955 & 45,570 \\
\hline Range & $2,542-399,960$ & $2,236-399,960$ & $2,068-399,960$ \\
\hline \multicolumn{4}{|l|}{ Reported symptoms } \\
\hline History of fever $(\%)$ & $116 / 117(99.2)$ & 115/115 (100) & $118 / 119(99.2)$ \\
\hline Anorexia (\%) & 24/117 (20.5) & 21/114 (18.4) & 19/119 (16.0) \\
\hline Vomiting (\%) & $34 / 117(29.1)$ & $32 / 115(27.8)$ & 25/119 (21.0) \\
\hline Diarrhea $(\%)$ & $5 / 117(4.3)$ & $3 / 116(2.6)$ & $3 / 119(2.5)$ \\
\hline Difficulty in breathing (\%) & $17 / 117(14.5)$ & $16 / 115(13.9)$ & $15 / 119(12.6)$ \\
\hline Lethargy (\%) & 9/117 (7.7) & $7 / 115(6.1)$ & $9 / 119(7.6)$ \\
\hline $\operatorname{Rash}(\%)$ & 4/117 (3.4) & $6 / 115(5.2)$ & $6 / 118(5.1)$ \\
\hline Unable to sit/stand as normal (\%) & 2/113 (1.8) & $4 / 112(3.6)$ & $5 / 116(4.3)$ \\
\hline
\end{tabular}

Figures in brackets are $95 \%$ confidence intervals unless otherwise specified.

$\mathrm{AQ}$, amodiaquine; SP, sulfadoxine-pyrimethamine; PCV, packed cell volume. 
TABLE 2

Safety evaluation

\begin{tabular}{|c|c|c|c|}
\hline Variable & $\mathrm{AQ}(\mathrm{n}=118)$ & $\mathrm{SP}(\mathrm{n}=117)$ & $\mathrm{AQ}+\mathrm{SP}(\mathrm{n}=119)$ \\
\hline \multicolumn{4}{|l|}{ Clinical } \\
\hline Death & 0 & 1 & 0 \\
\hline Total admissions & 16 & 10 & 6 \\
\hline Mean follow-up day admitted & 14.0 & 11.4 & 10.6 \\
\hline Total outpatient visits & 42 & 50 & 43 \\
\hline Rash & 6 & 5 & 7 \\
\hline \multicolumn{4}{|c|}{ Numbers developing new symptoms* (\%) } \\
\hline Anorexia & 41/93 (44.1) & 41/93 (44.1) & $47 / 100(47.0)$ \\
\hline Vomiting $t$ & $14 / 83(16.9)$ & $27 / 83(32.5)$ & $29 / 94(30.8)$ \\
\hline Diarrhea & $38 / 112(33.9)$ & $32 / 113(28.3)$ & $28 / 116(24.1)$ \\
\hline Breathing problems $\ddagger$ & $9 / 100(9.0)$ & $17 / 99(17.2)$ & $10 / 104(9.6)$ \\
\hline Lethargy & $39 / 108(36.1)$ & $33 / 108$ (30.6) & $24 / 110(21.8)$ \\
\hline Rash & $6 / 113(5.3)$ & $4 / 109(3.7)$ & $5 / 112(4.5)$ \\
\hline Convulsions & $0 / 117$ & $0 / 116$ & $1 / 119(0.8)$ \\
\hline Unable to sit/stand as normal & $14 / 111(12.6)$ & 15/108 (13.9) & $8 / 111(7.2)$ \\
\hline \multicolumn{4}{|l|}{ Hematology§ (\%) } \\
\hline Total leukocytes $<5,000 / \mu \mathrm{L}$ & $3 / 81(3.7)$ & $3 / 90(3.3)$ & 2/94 (2.1) \\
\hline Lymphocytes $<4.0$ & $29 / 81(35.8)$ & $26 / 90(28.9)$ & $19 / 94(20.2)$ \\
\hline Neutrophils $<1.5$ & $2 / 81(2.5)$ & $1 / 90(1.1)$ & $2 / 94(2.1)$ \\
\hline Platelets $<100$ & $3 / 81(3.7)$ & $6 / 90(6.7)$ & $5 / 94(5.3)$ \\
\hline $\mathrm{PCV}<33 \%$ & $29 / 81(35.8)$ & $30 / 90(33.3)$ & $23 / 94(24.5)$ \\
\hline \multicolumn{4}{|l|}{ Biochemistryq } \\
\hline $\mathrm{ALT}>40$ & $2 / 66(3.0)$ & $1 / 72(1.4)$ & $1 / 81(1.2)$ \\
\hline$\gamma-\mathrm{GT}>40$ & $4 / 59(6.8)$ & $1 / 60(1.7)$ & $1 / 71(1.4)$ \\
\hline Creatinine $>130$ & $0 / 71(0)$ & $0 / 77(0)$ & $0 / 84(0)$ \\
\hline
\end{tabular}

AQ, amodiaquine; SP, sulfadoxine-pyrimethamine; PCV, packed cell volume; ALT, alanine transaminase; $\gamma$-GT, $\gamma$-glutamyl transferase.

* Denominators for symptom reports are number of children without the symptom at recruitment.

$\dagger \chi_{2}^{2} 6.3, P=0.04$ (AQ vs SP).

$\ddagger \chi^{2} 2.9, P=0.08$ (AQ vs SP)

\$ Haemtaology cut-off values taken from Practical Haematology, Danci JV \& Lewis SM, 8th Ed, 1995, Churchill Livingstone.

पI Biochemistry cut-off values provided by biochemistry laboratory.

later the same day. The clinical assessment was a progression of her clinical malaria rather than an adverse reaction to SP.

More children were admitted after treatment with AQ alone than children treated with SP or AQ+SP. These admissions tended to be later during the course of follow-up and were mostly $(14 / 16$, or $87.5 \%)$ due to malaria. Hence, these admissions reflect low efficacy of AQ rather than adverse responses to the treatment. The total number of outpatient attendances was similar in all groups, as was the frequency of any skin rash. The 14 non-scabetic rashes were all asymptomatic macular-papular eruptions noticed incidentally at presentation.

More children receiving SP reported vomiting during the course of follow-up than children receiving AQ alone (see Table 2). There was also a suggestion that children receiving SP alone were more likely to give a history of breathing difficulties during the course of follow-up than children receiving $\mathrm{AQ}$ or $\mathrm{AQ}+\mathrm{SP}$. There were no significant differences in the proportions of children with abnormal hematological or biochemical test results on Day 28 (see Table 2).

Clinical efficacy (Table 3). Clinical outcomes in the three groups were similar in the first 7 days of follow-up. There were no late treatment failures in either the SP or $\mathrm{AQ}+\mathrm{SP}$ groups. However, there were a considerable number of late treatment failures among children treated with AQ alone, particularly after Day 14 and reaching 27\% by the end of follow-up. By Day 21 children treated with AQ+SP were significantly less likely to require rescue medication than those treated with AQ alone, and by Day 28 children treated with SP alone were also significantly less likely to require rescue medication than those in the AQ group. There were no differences in the prevalence of severe anemia ( $\mathrm{PCV}<25 \%)$ at the end of follow-up (AQ 3/80, SP 4/89, AQ+SP 3/94: $\chi^{2}=$ $0.2, P=0.9)$.

Children who received SP alone were significantly more likely to be febrile (axillary temperature $\geq 37.5^{\circ} \mathrm{C}$ ) at each contact up to and including Day $7(P<0.01$ at each contact; Figure 2). By Day 21 children treated with AQ alone were more likely to be febrile $(5 / 77)$ than those treated with AQ+SP (0/80; Fisher's exact test $P=0.027)$. Analysis of the time taken for the other symptoms reported at recruitment (see Table 1) to resolve suggested no other differences between the treatment regimens.

Parasitological efficacy (Table 4). None of the 13 ETFs fulfilled criteria for R1-3 resistance and were excluded from the analysis of parasitological efficacy. In the remainder, the rate of parasite clearance was similar in the three groups over the first 3 days (see Figure 3), although children who received SP

TABLE 3

Cumulative clinical failure rates

\begin{tabular}{ccll}
\hline Day & AQ & SP & AQ+SP \\
\hline $1-3$ & $5 / 111$ & $5 / 108$ & $3 / 111$ \\
7 & $6 / 107$ & $5 / 101$ & $3 / 103$ \\
14 & $9 / 97$ & $5 / 89$ & $3 / 88$ \\
21 & $18 / 91^{*}$ & $5 / 81^{*}$ & $3 / 83$ \\
28 & $25 / 91 \dagger$ & $5 / 79 \dagger$ & $3 / 78$ \\
\hline
\end{tabular}

$\mathrm{AQ}$, amodiaquine; $\mathrm{SP}$, sulfadoxine-pyrimethamine

$\begin{aligned} * \chi_{2}^{2} & =6.85, P=0.009 \\ \dagger \chi^{2} & =13.01, P=0.0003\end{aligned}$ 


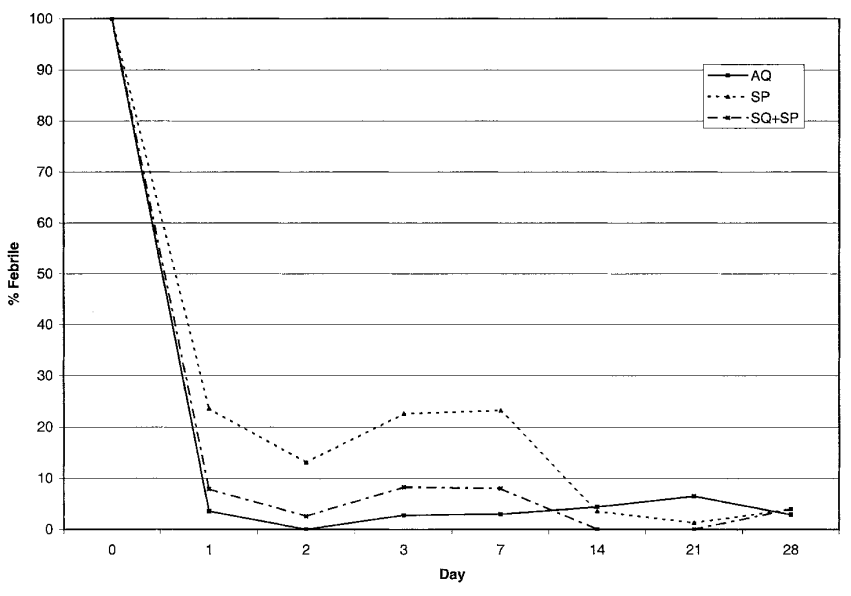

FIGURE 2. Fever clearance time.

alone were significantly less likely to be parasitemic on Day 2 than children in the other groups $(P<0.01)$. Overall levels of R-I resistance were considerably higher in children treated with AQ than in those receiving the other treatments, although the level of R-I resistance to SP is notable.

Only three children were gametocytemic at recruitment $(\mathrm{AQ}=1, \mathrm{AQ}+\mathrm{SP}=2)$. Of the 77 children with a $\mathrm{PCV}<25 \%$ at recruitment, $24(31.2 \%)$ became gametocytemic compared with $12(11.1 \%)$ of the 108 children with a PCV $\geq 33 \%$ at this time $\left(\chi^{2}=11.4, P=0.003\right)$. In comparison with children receiving $\mathrm{AQ}+\mathrm{SP}$, those treated with $\mathrm{SP}$ alone were more likely to be gametocytemic during follow-up, and these gametocytes tended to arise later during the course of follow-up (see Figure 4).

\section{DISCUSSION}

We have documented the safety and efficacy of AQ, SP, and $\mathrm{AQ}+\mathrm{SP}$ in the treatment of uncomplicated $P$. falciparum malaria in young children in southern Tanzania. The study was based on the standard WHO in vivo test protocol with

TABLE 4

Proportion of parasitological failures at days 7, 14, 21, and 28

\begin{tabular}{|c|c|c|c|}
\hline Variable & Sensitive & R-I & R-II \\
\hline \multicolumn{4}{|l|}{ Day 7} \\
\hline ÅQ $(\mathrm{n}=103)$ & $80(77.7 \%)$ & $6(5.8 \%)$ & $17(16.5 \%)$ \\
\hline $\mathrm{SP}(\mathrm{n}=99)$ & $74(74.8 \%)$ & $13(13.1 \%)$ & $12(12.1 \%)$ \\
\hline$A Q+S P(n=102)$ & $84(82.4 \%)$ & $9(8.8 \%)$ & $9(8.8 \%)$ \\
\hline \multicolumn{4}{|l|}{ Day 14} \\
\hline AQ (n = 97) & $66(68.0 \%)$ & $14(14.4 \%)$ & $17(17.5 \%)$ \\
\hline $\mathrm{SP}(\mathrm{n}=90)$ & $62(68.9 \%)$ & $16(17.8 \%)$ & $12(13.3 \%)$ \\
\hline$A Q+S P(n=89)$ & $68(76.4 \%)$ & $12(13.5 \%)$ & $9(10.1 \%)$ \\
\hline \multicolumn{4}{|l|}{ Day 21} \\
\hline $\mathrm{AQ}(\mathrm{n}=95)$ & $46(48.4 \%)$ & $32(33.7 \%)^{*}$ & $17(17.9 \%)$ \\
\hline $\mathrm{SP}(\mathrm{n}=84)$ & $54(64.3 \%)$ & $18(21.4 \%)^{*}$ & $12(14.3 \%)$ \\
\hline $\mathrm{AQ}+\mathrm{SP}(\mathrm{n}=84)$ & $60(71.4 \%)$ & $15(17.9 \%)$ & $9(10.7 \%)$ \\
\hline \multicolumn{4}{|l|}{ Day 28} \\
\hline AQ (n = 97) & $36(37.1 \%)$ & $44(45.4 \%) \dagger$ & $17(17.5 \%)$ \\
\hline $\mathrm{SP}(\mathrm{n}=82)$ & $49(59.8 \%)$ & $21(25.6 \%) \dagger$ & $12(14.6 \%)$ \\
\hline $\mathrm{AQ}+\mathrm{SP}(\mathrm{n}=81)$ & $52(64.2 \%)$ & $20(24.7 \%)$ & $9(11.1 \%)$ \\
\hline
\end{tabular}

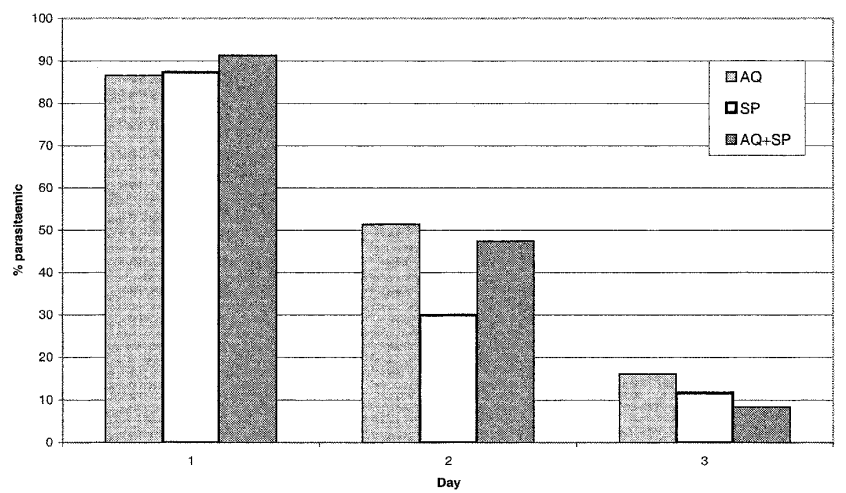

FIGURE 3. Resolution of parasitemia over Days 1-3. Day 2: SP $29.9 \%(32 / 107)$ versus AQ $51.4 \%(56 / 109) \chi^{2}=10.3, P=0.001$.

follow-up to 28 days. The number of children lost to follow-up and the reason-specific rates were comparable between the groups. Treatment with AQ alone produced good parasitological (78\%) and clinical (94\%) responses in the first 7 days and was associated with a shorter fever clearance time than SP alone. However, by the end of follow-up, AQ-only recipients were subject to a markedly higher level of R-I type resistance than children in the other treatment groups and were at least five times more likely to require rescue medication. These findings are striking in an area where AQ has not previously been readily available but where a 7-day parasitological cure rate of only $35 \%$ was documented for CQ. ${ }^{20}$ Concerns over cross-resistance between $\mathrm{CQ}$ and $\mathrm{AQ}$ seem to be legitimate, although the results of molecular studies will be needed to distinguish recrudescences from reinfections. Interestingly, anecdotal reports during the trial suggested that the faster symptom resolution associated with AQ gave an impression of it being a "stronger" drug, and mothers specifically requested retreatment with AQ. Such perceptions could be an important factor in the successful implementation of treatment policies. $^{23}$

The only death in the study cohort was a child who received SP alone and was due to progression of the malarial disease. It is not possible to say whether treatment with a faster-acting antimalarial would have altered the outcome for this child. Although the overall clinical cure rate for SP was good $(94 \%)$, it was associated with more vomiting than AQ alone and its slower onset of action was reflected by a prolonged

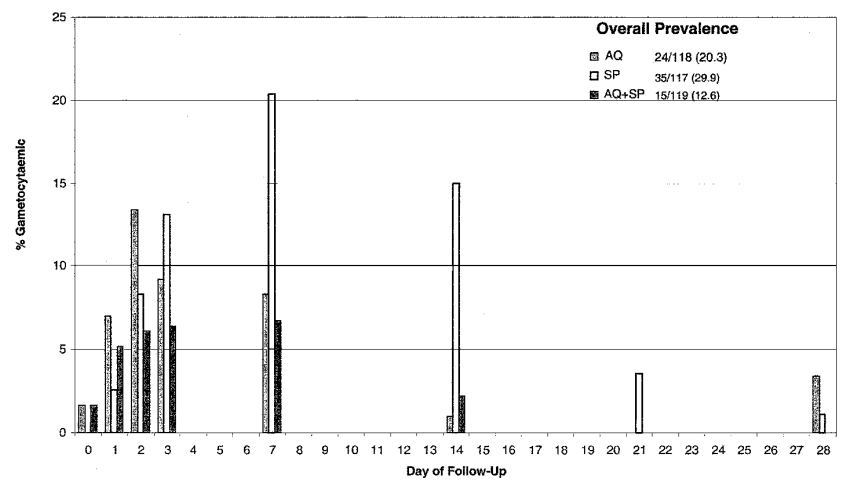

FIGURE 4. Gametocyte prevalence during follow-up. AQ versus SP: $\chi^{2}=2.9, P=0.09$. SP versus SP+AQ: $\chi^{2}=10.6, P=0.001$. 
fever clearance time. The parasitological profile after treatment with SP is also cause for concern. An R-I resistance level of $26 \%$ and an R-II level of $15 \%$ are bad news at a time when SP drug pressure is likely to increase dramatically as a result of the change in the national malaria treatment policy. Based on experience from elsewhere, ${ }^{10-12}$ it is likely that clinical resistance to SP will develop rapidly. This is of particular concern because several studies of CT include SP as a constituent drug; consequently, it may be that by the time SPcontaining CT regimens are available, their effectiveness will have been undermined by increased levels of SP resistance. More importantly, approaches to malaria control that require long half-life drugs, such as intermittent treatment in infants, ${ }^{24}$ may also be compromised.

The coadministration of $\mathrm{AQ}+\mathrm{SP}$ appeared to combine the rapid symptomatic relief associated with $\mathrm{AQ}$ with the greater longer-term efficacy of SP. However, it is not clear whether regular use of antipyretics during the first 2-3 days after presentation would be as effective in resolving symptoms as treatment with amodiaquine. The clinical and parasitological profiles after treatment with $\mathrm{AQ}+\mathrm{SP}$ were similar to those after treatment with SP alone.

Assessment of safety depended on data from outpatient attendances, admissions to hospital, systematically collected data on reported symptoms, and the results of hematological and biochemical testing. More weight should be given to the admission and outpatient department visit data than the reported symptom data because the latter was more often unavailable for children who were admitted than for children who were not, and these children were clearly more likely to be symptomatic. The data presented are thus likely to underestimate the prevalence of symptoms in children receiving only AQ. The higher rate of admission in the AQ-only group reflected its antimalarial efficacy rather than adverse reactions to the drug per se. Similarly, the increased tendency to give a history of breathing difficulties in children who received SP is likely to be a reflection of its slower onset of action compared with AQ. Hence, no drug-related severe adverse reactions were documented in any treatment group. It was particularly reassuring that there was no suggestion of increased frequencies of rash or bone marrow suppression in children receiving $\mathrm{AQ}+\mathrm{SP}$.

Children who were anemic at baseline were more likely to develop gametocytemia, in keeping with the findings from another setting 25 and presumably reflecting either a survival strategy of $P$. falciparum or a longer period of $P$. falciparum infection. Assuming the infectivity of gametocytes appearing after treatment with SP is $40 \%$ and after AQ it is $60 \%,{ }^{26}$ the data suggest that treatment of 100 individuals with SP would result in 12 infectious individuals compared with 13 with $A Q$ and 5 with AQ/SP. This extrapolation merits further investigation through mosquito-feeding assays, although its relevance to the intensity of transmission in an area would depend on the malaria treatment rates in the group of individuals constituting the main reservoir of infection.

The evolution and dynamics of resistance to antimalarials require new strategic thinking. Monotherapy with SP may not be an optimal choice for first-line treatment of malaria in endemic settings: the long half-life will tend to lead to the development of resistance more rapidly than shorter half-life drugs, reducing the effective life span of treatment policies depending on it. However, as resistance to chloroquine in- creases, National Malaria Control Programs are required to implement policies with cheap, available, and effective antimalarial drugs: SP is one of a very few alternatives. We have shown that significant levels of resistance to AQ exist in an area historically devoid of AQ but with high levels of CQ resistance, and that there are considerable levels of parasitological resistance to SP. The combination of $\mathrm{AQ}+\mathrm{SP}$ appeared to be safe and combined the benefits of rapid symptom relief with an improved parasitological response. An interim policy in which $\mathrm{AQ}+\mathrm{SP}$ was recommended would combine the treatment benefits of the combination and might reduce the rate at which SP resistance develops. Both AQ and SP are readily available and thus not subject to the likely time constraints of implementing a policy where SP is coadministered with artemether. Furthermore, these drugs are cheap; a treatment course of AQ costs TSh 23 (U.S. \$0.01) and a course of SP about TSh $11 .^{27,28}$ Finally, by reducing the rate at which resistance develops to SP, coadministration of $\mathrm{AQ}+\mathrm{SP}$ may protect SP for use as intermittent treatment and buy time while alternative, preferably short half-life, antimalarial treatments are evaluated for inclusion alongside artemether in malaria CT regimens.

Acknowledgments: We thank the mothers and the MCH clinic staff for their cooperation, Dr. P. Kibatala and Dr. F. Lwilla for their support, and the field workers, led by Morsad Nywage, for the extraordinary lengths they went to maximize follow-up rates.

Financial support: This investigation received financial support from the UNDP/World Bank/World Health Organization Special Programme for Research and Training in Tropical Diseases (TDR) and the Spanish Agency for International Co-operation (AECI). IHRDC receives core funding from the Swiss Agency for Development and Co-operation.

Authors' addresses: David Schellenberg, John J. Aponte, Clara Menendez, and Pedro L. Alonso, Unidad de Epidemiologia, Hospital Clinic, Barcelona, Spain, Telephone: +349-3227-5706, Fax: 349-34515272. Elizeus Kahigwa, Chris Drakeley, Athumani Malende, John Wigayi, Chris Msokame, and Hassan Mshinda, Ifakara Health Research and Development Centre, Ifakara, Tanzania, Telephone: +255-232-625-164, Fax: +255-232-625-312. Marcel Tanner, Swiss Tropical Institute, Basel, Switzerland, Telephone: +41-61-284-8427.

Reprint requests: Dr P L Alonso, Unidad de Epidemiologia, Hospital Clinic, Villaroel 170, Barcelona 08036, Spain. Tel + 3493227 5706, Fax: + 3493451 5272, E-mail: alonso@medicina.ub.es

\section{REFERENCES}

1. Snow RW, Craig M, Deichmann U, Marsh K, 1999. Estimating mortality, morbidity and disability due to malaria among Africa's non-pregnant population. Bull WHO 77: 624.

2. World Health Organization, 1993. Implementation of the Global Malaria Control Strategy. Report of a WHO study group on the implementation of the global plan of action for malaria control 1993-2000 (WHO technical report series 839). Geneva: WHO.

3. Trape JF, 1998. Impact of chloroquine resistance on malaria mortality. C R Acad Sci III 321: 689.

4. White NJ, Nosten F, Looaresuwan S, Watkins WM, Marsh K, Snow RW, Kokwaro G, Ouma J, Hien TT, Molyneux ME, Taylor TE, Newbold CI, Ruebush TK, Danis M, Greenwood BM, Anderson RM, Olliaro P, 2000. Averting a malaria disaster. Lancet 353: 1965.

5. von Seidlein L, Milligan P, Pinder M, Bojang K, Anyalebechi C, Gosling R, Coleman R, Ude JI, Sadiq A, Duraisingh M, Warhurst D, Alloueche A, Targett G, McAdam K, Greenwood B, Walraven G, Olliaro P, Doherty T, 2001. Efficacy of artesunate plus pyrimethamine-sulphadoxine for uncomplicated malaria in Gambian children: a double-blind, randomised, controlled trial. Lancet 355: 352-357. 
6. Kenyan Ministry of Health, 1998. National guidelines for diagnosis, treatment and prevention of malaria for health workers. Nairobi: Ministry of Health.

7. Malawi Ministry of Health, 1994. Guidelines for the management of malaria for medical officers, clinical officers, medical assistants and nursing staff (4th ed). Lilongwe, Malawi: Ministry of Health and Population.

8. Watkins WM, Msobo M, 1993. Treatment of Plasmodium falciparum malaria with pyrimethamine-sulfadoxine: selective pressure for resistance is a function of long elimination halflife. Trans R Soc Trop Med Hyg 87: 75-78.

9. Nzila AM, Nduati E, Mberu EK, Hopkins Sibley C, Monks SA, Winstanley PA, Watkins WM, 2000. Molecular evidence of greater selective pressure for drug resistance exerted by the long-acting antifolate pyrimethamine/sulfadoxine compared with the shorter-acting chlorproguanil/dapsone on Kenyan Plasmodium falciparum. J Infect Dis 181: 2023-2028.

10. Ronn AM, Msangeni HA, Mhina J, Wernsdorfer WH, Bygbjerg IC, 1996. High level of resistance of Plasmodium falciparum to sulfadoxine-pyrimthamine in children in Tanzania. Trans $R$ Soc Trop Med Hyg 90: 179-181.

11. van Dillen J, Custers M, Wensink A, Wouters B, van Voorthuizen T, Voorn W, Khan B, Muller L, Nevill C, 2000. A comparison of amodiaquine and sulfadoxine-pyrimethamine as first-line treatment of falciparum malaria in Kenya. Trans $R$ Soc Trop Med Hyg 93: 185-188.

12. White N, 1992. Antimalarial drug resistance: the pace quickens. $J$ Antimicrob Chemother 30: 571-585.

13. Olliaro P, Nevill CG, Ringwald P, Mussano P, Garner P, Brasseur $P, 1996$. Systematic review of amodiaquine treatment in uncomplicated malaria. Lancet 348: 1196-1201.

14. Duraisingh MT, Drakeley CJ, Muller O, Bailey R, Snounou G, Targett GA, Greenwood BM, Warhurst DC, 1997. Evidence for selection for the tyrosine-86 allele of the pfmdr 1 gene of Plasmodium falciparum by chloroquine and amodiaquine. Parasitology 114: 205-211.

15. National Malaria Control Program, Ministry of Health, 2000. Malaria treatment guidelines. Dar es Salaam, Tanzania: Ministry of Health.

16. Bojang KA, Palmer A, van Hensbroek MB, Banya WAS, Greenwood BM, 1997. Management of severe malarial anaemia in Gambian children. Trans Roy Soc Trop Med 91: 557-561.

17. McIntosh HM, Greenwood BM, 1998. Chloroquine or amodiaquine combined with sulfadoxine-pyrimethamine as a treat- ment for uncomplicated malaria-a systematic review. Ann Trop Med Parasitol 93: 265-270.

18. Bjorkman A, Phillips-Howard PA, 1991. Adverse reactions to sulpha drugs: implications for malaria chemotherapy. Bull WHO 69: 297-304.

19. Menendez C, Kahigwa E, Hirt R, Vounatsou P, Aponte JJ, Font F, Acosta CJ, Schellenberg DM, Galindo CM, Kimario J, Urassa H, Brabin B, Smith TA, Kitua AY, Tanner M, Alonso PL, 1997. Randomised placebo-controlled trial of iron supplementation and malaria chemoprophylaxis for prevention of severe anaemia and malaria in Tanzanian infants. Lancet 350: 844-849.

20. Hatz C, Abdullah S, Mull R, Schellenberg D, Gathmann I, Kibatala P, Beck H, Tanner M, Royce C, 1998. Efficacy and safety of CGP56697 (artemether and benflumetol) compared with chloroquine to treat acute falciparum malaria in Tanzanian children aged 1-5 years. Trop Med Int Health 3: 498-504.

21. World Health Organization, 1996. Assessment of therapeutic efficacy of antimalarial drugs. For uncomplicated falciparum malaria in areas with intense transmission. WHO 1996/MAL/961077.

22. Rieckmann KH, 1990. Monitoring the response of malaria infections to treatment. Bull WHO 68: 759-760.

23. Williams HA, Kachur SP, Nalwamba NC, Hightower A, Simoonga C, Mphande PC, 1999. A community perspective on the efficacy off malaria treatment options for children in Lundazi District, Zambia. Trop Med Int Health 4: 641-652.

24. Schellenberg D, Menendez C, Kahigwa E, Aponte J, Vidal J, Tanner M, Mshinda H, Alonso P, 2001. Intermittent treatment for malaria and anaemia control at time of routine vaccinations in Tanzanian infants: a randomised, placebo-controlled trial. Lancet 357: 1471-1477.

25. Drakelety CJ, Secka I, Correa S, Greenwood BM, Targett GAT, 2000. Host haematological factors influencing the transmission of Plasmodium falciparum gametocytes to Anopheles gambiae S.S. mosquitoes. Trop Med Int Health 4: 131-138.

26. Robert V, Awono-Ambene HP, Le Hesran JY, Trape JF, 2000. Gametocytemia and infectivity to mosquitoes of patients with uncomplicated Plasmodium falciparum malaria attacks treated with chloroquine or sulfadoxine plus pyrimethamine. Am $J$ Trop Med Hyg 62: 210-216.

27. Management Sciences for Health, 1998. International drug price indicator guide. Boston: Management Sciences for Health.

28. Medical Stores Department, 1999. Essential drugs and medical supplies. Dar es Salaam, Tanzania: Medical Stores Department. 